\title{
Depression and borderline personality disorder
}

$\mathrm{B}$ orderline personality disorder (BPD) is a serious mental illness characterised by dysregulation of emotions and impulses, an unstable and inconsistent sense of self and of others in close relationships, and marked difficulties in interpersonal relationships, often accompanied by suicidal and selfharming behaviour.

The instability of emotions and predominance of negative affect that characterise BPD often lead to problems determining whether the patient has major depression co-occurring with $\mathrm{BPD}$, or whether the depressive symptoms are part and parcel of the BPD itself. In this article, we aim to assist clinicians facing this situation to make an accurate diagnosis.

To prepare the article, we searched PsychInfo and MEDLINE databases for articles published between 2000 and 2012 relating to BPD co-occurring with major depression, other depressive disorders, or bipolar disorder. Review articles and those involving randomised controlled trials of treatment were particularly sought. Book chapters relevant to the search criteria were also examined.

\section{The diagnostic problem}

Major depressive disorder (MDD) commonly co-occurs with BPD. The lifetime prevalence of major depression in the course of BPD was $83 \%$ in one large study, ${ }^{1}$ which accords with other research and clinical experience.

Patients with BPD often present to clinicians with depressive symptoms. As the symptoms of depression and BPD overlap significantly, it can be challenging to make an accurate diagnosis of a major depressive illness when the disorders co-occur. Accurate diagnosis is essential because each disorder requires treatment in its own right. It is important to note that rating scales of depression, whether patient- or clinician-rated, are less helpful for assessing the severity of depressive symptoms when BPD is present. ${ }^{2}$

\section{BPD is not a variant of affective disorder}

Given the prominent overlap of symptoms between BPD and affective disorders, it has been suggested that BPD is a variant of affective disorder - either depressive disorder or bipolar disorder. ${ }^{3,4}$ However, the consensus of expert opinion is that BPD is not a variant of either MDD or bipolar disorder, ${ }^{5-12}$ although overlap of symptoms of both disorders can occur with BPD.

The most significant evidence that BPD is not a variant of depressive disorder is that treatment of depression does not result in remission of BPD symptoms. An important longitudinal study found that effective treatment of BPD tends to result in remission of depression, and antidepressants often show only modest benefit for depressive disorders that co-occur with BPD. ${ }^{6}$

A 2010 review of phenotype, endophenotype and genotype comparisons between BPD and MDD found that

\section{Summary}

- Borderline personality disorder (BPD) is a serious mental illness characterised by dysregulation of emotions and impulses, an unstable sense of self, and difficulties in interpersonal relationships, often accompanied by suicidal and self-harming behaviour.

- Major depressive disorder (MDD) commonly co-occurs with BPD. Patients with BPD often present with depressive symptoms.

- It can be difficult to distinguish between BPD and MDD, especially when the two disorders co-occur.

- Research is needed to clarify the commonalities and differences between BPD and MDD, and BPD and rapid-cycling bipolar disorder.

- When MDD and BPD co-occur, both conditions should be treated concurrently.

- MDD co-occurring with BPD does not respond as well to antidepressant medication as MDD in the absence of BPD.

- MDD is not a significant predictor of outcome for BPD, but BPD is a significant predictor of outcome for MDD.

- Treatment of BPD with specific psychotherapies tends to result in remission of co-occurring MDD.

- Empirically validated psychotherapies for BPD share common features that are applicable in all treatment settings where patients with BPD are likely to present, including primary care.

- Methodologically sound research is required to examine the effectiveness of medications for treatment of MDD co-occurring with BPD.

BPD differs from MDD in symptomatology, prognosis, heritability, patterns of brain region involvement, neurohormonal indices and sleep architecture. ${ }^{7}$ Some biological processes overlapped, including amygdala hyperreactivity, volume changes in the anterior cingulate cortex, and deficient serotonin function. The authors noted that definitive clarification of the commonalities and differences between BPD and MDD requires examination of both disorders using the same study design and methodology.

A study of depressive symptoms and BPD features in dysthymic disorder showed that a common factor underlying both disorders best explained the frequency of their co-occurrence, providing an excellent fit with the data. ${ }^{13}$ The authors postulated that the underlying factors were shared genetic, temperamental, or early environmental risk factors.

It is known that factors in the early environment, including those that lead to insecure and pathological patterns of attachment, combined in some cases with an anxious, sensitive temperament and later childhood trauma, predispose to both BPD and early-onset dysthymic disorder and depression. ${ }^{14}$ It may be that some of these factors also predispose to rapid-cycling bipolar disorder. 
Family studies show that, while MDD and bipolar disorders commonly co-occur with BPD, impulsive spectrum disorders are more common than affective spectrum disorders in BPD-affected families. ${ }^{10,11}$ Studies have also shown that depressed patients with BPD do not significantly differ from those without BPD in terms of morbid risk for bipolar disorder in first-degree relatives. ${ }^{10,11}$ Importantly, treatment with mood-regulating agents does not produce remission from BPD. ${ }^{10}$

A recent review examining the similarities and differences between bipolar affective disorder and BPD concluded that they are separate entities but have significant elements in common. ${ }^{12}$ The many differences between them included those relating to sense of self, relationship disruptions, family history of bipolar disorders, benefits of medication, and the form of affective dysregulation.

Another recent review of the overlap between bipolar disorder and BPD found the greatest overlap occurred in relation to rapid-cycling bipolar disorder. ${ }^{15}$ Frequent shifts of mood characterised both BPD and rapid-cycling bipolar disorder, as did a history of childhood trauma including sexual, physical and emotional abuse, and neglect. Maladaptive self-schemas of the "I am bad" type were much more characteristic of $\mathrm{BPD}$, as was vulnerability to abandonment. The review's authors question whether rapid-cycling bipolar disorder is a form of BPD or represents bipolar disorder with co-occurring BPD traits.

Clearly, there are unanswered questions about the reasons for the frequent co-occurrence of affective disorders and BPD, which can only be resolved by further research.

\section{Depressive symptoms in BPD in the absence of major depression}

Depressive symptoms that occur as part of BPD are usually transient and related to interpersonal stress (eg, after an event arousing feelings of rejection). Such "depression" usually lifts dramatically when the relationship is restored. Depressive symptoms in BPD may also serve to express feelings (eg, anger, frustration, hatred, helplessness, powerlessness, disappointment) that the patient is not able to express in more adaptive ways. The patient's "depression" in these cases represents a maladaptive endeavour to communicate his or her unhappiness about a particular person or situation. Such depressive states will not respond to antidepressant treatment, but to careful elucidation of the underlying feelings, followed by assisting the patient to address the problem in more adaptive ways.

On cross-sectional assessment, the transient depressive symptoms of BPD may be indistinguishable from symptoms of a major depressive episode (MDE). This can lead to incorrect diagnosis in the absence of a longitudinal history.

\section{Clarifying the presence of major depression in BPD}

A longitudinal history, with careful examination of the depressive symptoms over recent days and weeks, is required to make an accurate diagnosis of MDE or MDD co-occurring with BPD. The diagnostic criteria for MDE in patients with BPD are the same as those for MDE in the general population: consistently lowered mood for at least 2 weeks, significant impairment of energy, lowered interest in usual activities, sleep disturbance (which may involve insomnia or hypersomnia), weight loss or gain, increased suicidal ideation (sometimes accompanied by increased suicidal or other self-harming behaviour), anhedonia, sense of worthlessness, poor concentration and attention, and impairment of functioning (social, occupational or other). ${ }^{16}$ Melancholic and psychotic features are seldom seen in MDE co-occurring with BPD. However, although the overall pattern of symptoms is the same as in the general population, the quality of the depression in BPD is different. ${ }^{17-20}$ Depression in BPD is characterised by the features shown in Box 1.

It should be stressed that a diagnosis of MDE should be made in a patient with BPD only when the patient's mood has been consistently lowered for a minimum of 2 weeks, in conjunction with other symptoms sufficient to meet the criteria for MDE.

\section{Psychotropic medication for MDD co-occurring with BPD}

We are aware of no research specifically examining medication for major depression co-occurring with BPD. The consensus of informed opinion over many years has been that depression co-occurring with BPD does not respond as well to antidepressant medication as depression in the absence of BPD. ${ }^{5,21}$ In addition, there is evidence that the time to remission tends to be longer and recurrence of depression more likely when BPD and MDD co-occur. ${ }^{22} \mathrm{~A}$ meta-analysis of the outcome of depression co-occurring with personality disorders in general similarly concluded that: "Combined depression and personality disorder is associated with a poorer outcome than depression alone". ${ }^{23}$ The percentage of patients with BPD in the sample included in this analysis was not stated.

However, not all authorities agree that depression cooccurring with BPD responds poorly to antidepressant treatment. ${ }^{24,25}$ A 2002 review found that whether or not personality disorder worsened treatment outcome depended on the study design - the best-designed studies reported the least effect of personality disorder on the outcome of treatment for depression. ${ }^{25}$ Individual personality disorders were not examined separately. High neuroticism scores were found to be predictive of poor

1 Features of depression in borderline personality disorder

Feelings of loneliness, emptiness, boredom, alienation and intense sadness

- Clinging dependency, sense of desperation in relation to the absence of (or fear of rejection or abandonment by) significant others

- Instability of negative affect

- Deep sense of inner badness, with accompanying merciless attack on the self

- Increased suicidal ideation and behaviour

Rarity of melancholic symptoms 
prognosis, particularly when long-term outcome was taken into account. High neuroticism scores are characteristic of $\mathrm{BPD}^{26}$ and relapse of depression tends to be earlier and time of remission shorter in $\mathrm{BPD}^{22}$ suggesting that patients with BPD and co-occurring depression may fall in the group identified in this review as responding poorly to treatment for depression.

We agree that vigorous treatment of depression is required when it co-occurs with $\mathrm{BPD},{ }^{25}$ to ensure the best possible outcome for the patient, but believe that this must be combined with treatment for the co-occurring BPD. In the absence of adequate data, clinicians should consider treating MDD associated with BPD with biological treatments (antidepressants), as they would treat MDD without BPD. However, without BPD-specific psychotherapy, MDD that is associated with BPD may not respond adequately to biological treatments - but BPDspecific psychotherapy does help treat both MDD and BPD when the disorders co-occur.

There is some limited evidence for the use of aripiprazole, olanzapine and omega- 3 fatty acids in the management of depressive symptoms of $\mathrm{BPD},{ }^{21}$ but there are no data to guide clinicians in choosing a specific biological treatment for MDD that co-occurs with BPD. Lithium has not been shown to be particularly effective in treating MDD that co-occurs with BPD. Clinicians may need to exercise caution in prescribing lithium for patients with BPD and MDD, given the significant risk (about 25\%) of self-harm through overdose of prescription medication in patients with BPD. ${ }^{27}$

Rigorous research examining the treatment response of patients with BPD and co-occurring depression is clearly required and should include patients with severe forms of BPD, who are likely to be among those responding most poorly to treatment for co-occurring depression.

\section{Polypharmacy and BPD}

There is increasing pressure worldwide to limit the use of medication for BPD because of its limited effectiveness and concerns about the obesity-related health problems that can occur, particularly with polypharmacy. ${ }^{21}$ Guidelines published by the United Kingdom's National Institute for Health and Clinical Excellence in 2010 go so far as to state that drug treatment should not be used for individual symptoms or behaviour associated with $\mathrm{BPD}^{28}$ whereas the latest Cochrane review states that pharmacotherapy should be targeted at specific symptoms only. ${ }^{29}$ Both guidelines acknowledge the possible benefit of medication when depression and BPD co-occur, while stressing that a diagnosis of depression should be reviewed when it has been made in the context of a crisis in the patient's life.

Unfortunately, polypharmacy is commonly seen in patients with BPD, with or without co-occurring depression. It tends to occur because the patient's distress is of such intensity that clinicians feel driven to try to alleviate it, by whatever means are available. Such means often include increased doses of medication or additional medications. The ensuing danger is that patients with BPD may be prescribed one psychotropic agent after another, sometimes in high doses, with none of the earlier prescriptions ceased. One study found that, compared with people with major depression alone, people with BPD were twice as likely to have received anti-anxiety medication, more than six times as likely to have received mood stabilisers, more than 10 times as likely to have used antipsychotics, and twice as likely to have taken antidepressants. ${ }^{30}$

All available evidence points to the fact that medication should be targeted at specific symptoms of BPD and used only when these are severe, and that medications should be reviewed on a regular basis. ${ }^{21,28,29}$

\section{Electroconvulsive therapy for MDD co-occurring with BPD}

There is little evidence that electroconvulsive therapy (ECT) is beneficial for treating depression in patients with BPD. ${ }^{21}$ There are no randomised controlled trials of its use, and studies have in general been poorly designed. A 2004 study employing improved methodology continued to show a poorer acute response to ECT for depression COoccurring with BPD. ${ }^{31}$ However, if patients with BPD have severe depression that would otherwise warrant ECT, they should not be excluded from consideration of this intervention simply on the basis of having BPD.

\section{Prioritising psychosocial treatment for BPD co-occurring with MDD}

The principal treatment for BPD is psychosocial - that is, some form of psychotherapy, which may be combined with psychotropic medication aimed at specific symptoms. ${ }^{32,33}$

One study showed that MDD is not a significant predictor of outcome for BPD, but BPD is a significant predictor of outcome for MDD. ${ }^{6}$ This finding led the study authors to recommend that treatment of BPD with psychotherapy should take priority when BPD co-occurs with depression, as once the BPD abates, so will the depression. ${ }^{6}$

Several modalities of psychotherapy for BPD, including dialectical behaviour therapy, mentalisation-based treatment, transference-focused therapy, schema-focused therapy and supportive psychotherapy, have been shown in randomised controlled trials to result in lowered levels of depression. ${ }^{33}$ It is unclear whether the depression referred to in these studies is part of an MDE co-occurring with the BPD or the depressive symptoms so often seen in BPD. These psychotherapies share some common features that are applicable across all treatment settings where patients with BPD are likely to present, including primary care (Box 2). There is no doubt that interactions with patients with BPD that lack these core features will worsen their distress and can lead to increasingly maladaptive (including self-harming) behaviour.

It is significant that those psychotherapies that have proven useful for the treatment of depression alone cognitive behaviour therapy, mindfulness-based approaches, and interpersonal therapy, among others all focus on promoting the patient's capacity to reflect on his or her own mind and that of others (ie, to "mentalise"). ${ }^{34}$ In some cases, a lowered rate of relapse of depression after psychotherapy has been shown. This lower relapse rate is likely to result from the patient's new- 


\section{Core features of psychotherapies for borderline} personality disorder

- Engaging the patient with a supportive, empathic attitude

- A respectful, cooperative, collaborative, active, open and non-judgemental relationship with the patient

- Focusing on the patient's mind, rather than his or her behaviour, and seeking to help the patient understand what lies behind maladaptive behaviour

- Validation of the patient's distress (but not necessarily of the behaviour that has accompanied it)

- An atmosphere of hope and optimism

found ability to reflect on the thoughts, feelings and behaviour associated with, or leading to, depression. ${ }^{34}$

\section{Conclusions}

We suggest taking a therapeutic stance to MDD cooccurring with BPD that promotes reflection on the patient's mind and the minds of others, using a psychological approach such as cognitive behaviour therapy, mindfulness, interpersonal psychotherapy or mentalisation-based treatment. Such reflection can, over time, limit the repetition of the maladaptive ways of thinking, interacting and behaving that lead to depression and depressive symptoms in patients with BPD. This approach, combined with the features common to effective therapies for BPD shown in Box 2, will help patients to understand themselves and others better, and can lead to lasting change, including less depression.

Medication for MDE, when it co-occurs with BPD, should be prescribed with careful attention to the dangers of polypharmacy and in the knowledge that medication is not the primary treatment for BPD. Leading authorities stress that medication for depression co-occurring with BPD will not lead to remission of the personality disorder, ${ }^{6,21}$ which requires treatment in its own right.

Competing interests: No relevant disclosures.

Provenance: Commissioned by supplement editors; externally peer reviewed.

1 Zanarini MC, Frankenburg FR, Dubo ED, et al. Axis I comorbidity of borderline personality disorder. Am J Psychiatry 1998; 155: 1733-1739.

2 Skodol AE, Stout RL, McGlashan TH, et al. Co-occurrence of mood and personality disorders: a report from the Collaborative Longitudinal Personality Disorders Study (CLPS). Depress Anxiety 1999; 10: 175-182.

3 Akiskal HS, Chen SE, Davis GC, et al. Borderline: an adjective in search of a noun. J Clin Psychiatry 1985; 46: 41-48.

4 Akiskal HS. The bipolar spectrum - the shaping of a new paradigm in psychiatry. Curr Psychiatry Rep 2002; 4: 1-3.

5 Gunderson J. Borderline personality disorder: a clinical guide. 2nd ed. Washington, DC: American Psychiatric Publishing, 2008.

6 Gunderson JG, Morey LC, Stout RL, et al. Major depressive disorder and borderline personality disorder revisited: Iongitudinal interactions. J Clin Psychiatry 2004; 65: 1049-1056.

7 Goodman M, New AS, Triebwasser J, et al. Phenotype, endophenotype, and genotype comparisons between borderline personality disorder and major depressive disorder. J Pers Disord 2010; 24: 38-59.

8 Galione J, Zimmerman M. A comparison of depressed patients with and without borderline personality disorder: implications for interpreting studies of the validity of the bipolar spectrum. J Pers Disord 2010; 24: $763-772$

9 Beatson J.Borderline personality disorder: history of the borderline concept. In: Beatson J, Rao S, Watson C. Borderline personality disorder: towards effective treatment. Melbourne: Australian Postgraduate Medicine, 2010: 1-16.

10 Paris J. Borderline or bipolar? Distinguishing borderline personality disorder from bipolar spectrum disorders. Harv Rev Psychiatry 2004; 12 $140-145$

11 Gunderson JG, Weinberg I, Daversa MT, et al. Descriptive and longitudinal observations on the relationship of borderline personality disorder to bipolar disorder. Am J Psychiatry 2006; 163: 1173-1178.

12 Bassett D. Borderline personality disorder and bipolar affective disorder Spectra or spectre? A review. Aust N Z J Psychiatry 2012; 46: 327-339.

13 Klein DN, Schwartz JE. The relation between depressive symptoms and borderline personality disorder features over time in dysthymic disorder. J Pers Disord 2002: 16: 523-535.

14 Beatson J, Taryan S. Predisposition to depression: the role of attachment. Aust N Z J Psychiatry 2003; 37: 219-225.

15 Coulston CM, Tanious M, Mulder RT, et al. Bordering on bipolar: the overlap between borderline personality and bipolarity. Aust N Z J Psychiatry 2012; 46: 506-521.

16 American Psychiatric Association. Diagnostic and statistical manual of mental disorders, 4th ed (DSM-IV). Washington, DC: American Psychiatric Publishing, 1994.

17 Levy KN. Edell WS, McGlashan TH. Depressive experiences in inpatients with borderline personality disorder. Psychiatr Q 2007; 78: 129-143.

18 Paris J. Treatment of borderline personality disorder: a guide to evidence-based practice. New York: Guilford Press, 2008.

19 Westen D, Moses MJ, Silk KR, et al. Quality of depressive experience in borderline personality disorder and major depression: when depression is not just depression. J Pers Disord 1992; 6: 382-393.

20 Silk KR. The quality of depression in borderline personality disorder and the diagnostic process. J Pers Disord 2010; 24: 25-37.

21 Rao S. Biological treatments. In: Beatson J, Rao S, Watson C. Borderline personality disorder: towards effective treatment. Melbourne: Australian Postgraduate Medicine, 2010: 87-124.

22 Grilo CM, Stout RL, Markowitz JC, et al. Personality disorders predict relapse after remission from an episode of major depressive disorder: a 6-year prospective study. J Clin Psychiatry 2010; 71: 1629-1635.

23 Newton-Howes G, Tyrer $P$, Johnson T. Personality disorder and the outcome of depression: meta-analysis of published studies. $\mathrm{Br} J$ Psychiatry 2006; 188: 13-20.

24 Mulder RT, Joyce PR, Frampton CM. Personality disorders improve in patients treated for major depression. Acta Psychiatr Scand 2010; 122: 219-225.

25 Mulder R. Personality pathology and treatment outcome in major depression: a review. Am J Psychiatry 2002; 159: 359-371.

26 Widiger TA. A temperament model of borderline personality disorder. In: Zanarini MC, editor. Borderline personality disorder. New York: Taylor and Francis, 2005: 63-81.

27 Makela EH, Moeller KE, Fullen JE, Gunel E. Medication utilization patterns and methods of suicidality in borderline personality disorder. Ann Pharmacother 2006; 40: 49-52.

28 National Collaborating Centre for Mental Health. Borderline personality disorder: treatment and management. Clinical guideline no. 78. London: National Institute for Health and Clinical Excellence, 2009.

29 Lieb K, Völlm B, Rücker G, et al. Pharmacotherapy for borderline personality disorder: Cochrane systematic review of randomised trials. Br J Psychiatry 2010; 196: 4-12.

30 Bender DS, Dolan RT, Skodol AE, et al. Treatment utilization by patients with personality disorders. Am J Psychiatry 2001; 158: 295-302.

31 Feske U, Mulsant BH, Pilkonis PA, et al. Clinical outcome of ECT in patients with major depression and comorbid borderline personality disorder. Am J Psychiatry 2004; 161: 2073-2080.

32 American Psychiatric Association Practice Guidelines. Practice guideline for the treatment of patients with borderline personality disorder. Am J Psychiatry 2001; 158 (10 Suppl): 1-52.

33 Beatson J, Rao S. Psychotherapy for borderline personality disorder. In: Beatson J, Rao S, Watson C. Borderline personality disorder: towards effective treatment. Melbourne: Australian Postgraduate Medicine, 2010: 125-166.

34 Luyten P, Fonagy P, Lemma A, Target M. Depression. In: Bateman AW, Fonagy $P$, editors. Handbook of mentalizing in mental health practice Washington, DC: American Psychiatric Publishing, 2012: 385-417. 